\section{La experiencia de Perú con un programa nacional de bancos de sangre}

\author{
José Fuentes Rivera Salcedo ${ }^{1}$ y \\ Oscar Roca Valencia ${ }^{1}$
}

En el Perú, la sangre usada con fines terapéuticos no alcanzaba, hasta 1996, la calidad ni la seguridad que tenía la de otros países del continente y la Organización Panamericana de la Salud (OPS) la consideraba una de las más peligrosas en términos de la transmisión de infecciones transfusionales, siendo la tasa de infección de una por cada 81 transfusiones (1). Todo esto se debía a la carencia de una política nacional que estableciera las medidas y acciones destinadas a garantizar la seguridad y calidad de la sangre obtenida, procesada y utilizada. De hecho, los bancos de sangre se consideraban lugares de depósito o almacenamiento de sangre para su uso posterior en pacientes.

La situación presente en 1996 era insatisfactoria (2). Casi todas las donaciones se realizaban para reposición $(99,96 \%)$, muchos de los donantes eran vendedores consuetudinarios de su sangre, y solo $0,04 \%$ eran donantes voluntarios genuinos. Esto obedecía a que los bancos de sangre exigían a los pacientes que buscaran donantes y no tenían una estrategia de promoción como las de otros países. Esta situación, además, llevaba a que personas sin escrúpulos se dedicaran a comercializar la sangre, ya fuera directamente o como intermediarios de "donantes" que luego hacían el depósito o la "donación" de la sangre requerida.

Por otra parte, no existían normas que garantizaran o regularan la seguridad y calidad de la sangre obtenida ni el funcionamiento idóneo de los bancos de sangre. No fue hasta 1995 que se promulgó la Ley 26454, aunque las medidas iniciales para lograr su ejecución y cumplimiento no se pusieron en práctica de inmediato.

La mayoría de los bancos de sangre realizaban solo pruebas para el tamizaje de infecciones por el virus de la inmunodeficiencia humana (VIH), el de la hepatitis B y la bacteria causal de la sífilis. Sin embargo, esto no impedía que ocurrieran infecciones transfusionales $(50 \%$, hepatitis $\mathrm{B} ; 38 \%$, hepatitis C; y $25 \%$, enfermedad de Chagas).

La infraestructura y el equipamiento de los bancos de sangre eran deplorables. La mayoría de ellos solo contaban con una o dos habitaciones y un área total máxima de $20 \mathrm{~m}^{2}$. La sangre se almacenaba en refrigeradores domésticos y las unidades de plasma en sus congeladores, y estas medidas no ofrecían las garantías necesarias; solo unos pocos bancos de sangre (no más de 20 en todo el país) contaban con centrífugas refrigeradas y podían fraccionar la sangre. 
También era insuficiente la capacitación de recursos humanos. El personal que trabajaba en los bancos de sangre solo había recibido capacitación durante sus estudios universitarios o académicos y solo unos pocos trabajadores habían participado en cursos de capacitación, pero sin el apoyo de la institución donde trabajaban.

\section{EL PROGRAMA NACIONAL DE HEMOTERAPIA Y BANCOS DE SANGRE}

A raíz de la infección de una madre por una transfusión sanguínea contaminada con VIH, el Congreso de la República promulgó la Ley 26454, que declaraba actividad de orden público e interés nacional la donación, obtención, procesamiento, transfusión y distribución de la sangre; estableció los lineamientos generales para el funcionamiento de los bancos de sangre; y creó el Programa Nacional de Hemoterapia y Bancos de Sangre (PRONAHEBAS) como entidad encargada de regular y supervisar el funcionamiento de estos servicios.

El PRONAHEBAS es un órgano dependiente de la Dirección General de Salud de las Personas, del Ministerio de Salud, y busca abastecer oportunamente sangre y hemocomponentes de la más alta calidad, obtenidos mediante donaciones voluntarias, con el objetivo de disminuir la mortalidad generada por su carencia, especialmente en grupos de alto riesgo, como las embarazadas y los niños. Por su parte, la misión del Programa es garantizar el cumplimiento de las normas vigentes relacionadas con la donación, obtención, procesamiento, transfusión y suministro de sangre, mediante la vigilancia y supervisión de los bancos de sangre de manera continua.

El PRONAHEBAS se puso en marcha en 1997, dos años después de promulgada la Ley 26454. La primera medida establecida por su director general ese mismo año fue hacer obligatorio que todos los bancos de sangre aplicaran siete pruebas para el tamizaje de agentes infecciosos a la sangre de todo donante. Las siete pruebas eran para la detección de anticuerpos contra el virus de la hepatitis C, los virus linfotrópicos de células T humanas (HTLV-I y II), el antígeno nuclear del virus de la hepatitis B, así como de marcadores de la enfermedad de Chagas, además de las tres pruebas realizadas hasta entonces. Esta medida, además de garantizar la seguridad de la sangre transfundida, permitió realizar una vigilancia epidemiológica permanente de estas enfermedades infecciosas en la población. Asimismo, se adoptó una política destinada a fomentar la donación voluntaria de sangre mediante campañas en todo el país, con la participación de las autoridades locales y la comunidad.

\section{Actividades realizadas}

Después de realizar el diagnóstico de la situación de los bancos de sangre en Perú durante 1997, el PRONAHEBAS estructuró un plan operativo en el que se plasmaron los objetivos, estrategias y actividades que permitirían, a corto y mediano plazo, garantizar el uso de sangre segura y oportuna.

Promoción de la donación voluntaria. En todo producto elaborado, la calidad depende de la materia prima. Este principio obliga a los bancos de sangre a tomar medidas para propiciar la captación de sangre de mejor calidad - es decir, obtenida de donantes voluntarios (figura 1) - de forma regular mediante campañas periódicas de donación, y para facilitarles a los donantes la donación de acuerdo con el tiempo del que disponen y no en horarios fijos, tal como se hacía anteriormente.

Promulgación de normas de seguridad de la sangre. Como se expuso más arriba, el número de pruebas para el tamizaje de agentes infecciosos que debían aplicarse a todo candidato a donante de sangre se incrementó de 3 a 7, sumándose a otras dos pruebas que debían efectuarse en zonas con malaria (paludismo) y bartoneliasis (enfermedad de Carrión o "verruga peruana") endémicas (3). Asimismo, toda unidad de sangre o hemocomponente destinada a transfusión debía contar con el Sello Nacional de Calidad de Sangre que otorga el PRONAHEBAS exclusivamente, según lo establecido en la Ley, previo cumplimiento de las normas antes mencionadas y de otras establecidas con posterioridad.

FIGURA 1. Resultados positivos en el tamizaje de enfermedades transmitidas por sangre en donantes voluntarios y donantes de reposición. Perú, 1997-2001

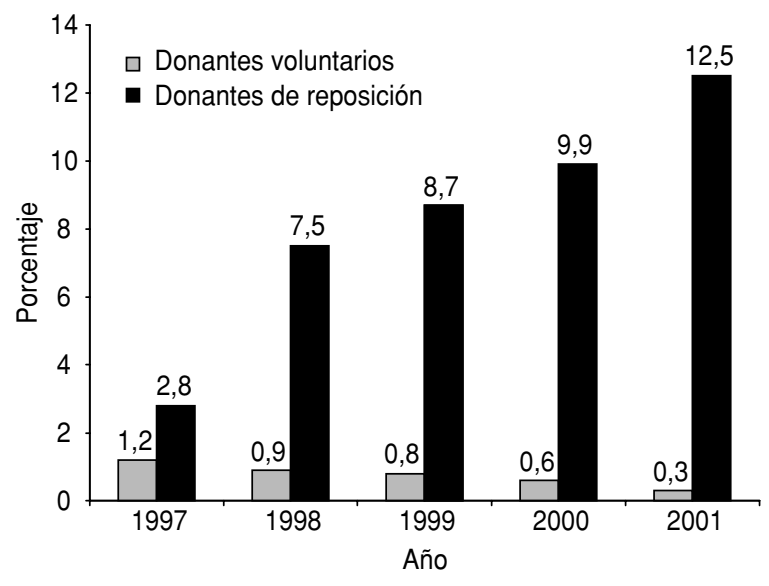


Capacitación del personal de bancos de sangre. El PRONAHEBAS implantó el curso a distancia "Sangre segura" mediante los módulos elaborados por la OPS sobre la materia. Inicialmente se evalúo de forma directa a los propios trabajadores de bancos de sangre que cumplieran determinados requisitos, entre ellos una evaluación por escrito de estos módulos y una pasantía o práctica profesional en bancos de sangre habilitados para entrenamientos de este tipo. Posteriormente, la capacitación se llevó a cabo en colaboración con la universidad, tal como en otros países como Ecuador y Colombia, mediante un diplomado en hemoterapia.

Elaboración de normas y reglamentos. Para cubrir los vacíos de la Ley 26454 se establecieron normas que brindaran el marco jurídico para el funcionamiento adecuado de los bancos de sangre; estimularan el uso racional de la sangre y los hemocomponentes por parte de los médicos, y fomentaran la donación voluntaria de sangre en Perú. Estas normas fueron promulgadas por el Ministerio de Salud mediante resoluciones ministeriales de cumplimiento obligatorio por todos los bancos de sangre, ya fueran públicos o privados.

Estandarización del funcionamiento de los bancos de sangre. Además de las normas promulgadas, se establecieron sistemas para estandarizar el trabajo en los bancos de sangre. Esto se logró mediante la capacitación de su personal, incluida la elaboración de manuales de normas, y procedimientos técnicos y administrativos, y mediante el desarrollo de un programa de control de calidad para validar la seguridad y calidad de la sangre.

\section{LOGROS OBTENIDOS}

Después de cinco años de trabajo se puede constatar que han mejorado considerablemente la seguridad, disponibilidad y calidad de la sangre en Perú. Se debe reconocer el esfuerzo desplegado por el personal de los bancos de sangre, buena parte del cual tuvo que afrontar desde limitaciones económicas hasta la incomprensión de las autoridades de salud para poder cumplir su labor, pues hasta ese momento se daba prioridad a otras necesidades y se restaba importancia a la disponibilidad de sangre debidamente procesada. Solo se pensaba en ello cuando se presentaba la necesidad, y en esos casos se violaban las normas jurídicas vigentes en aras de cubrir la emergencia existente. Asimismo, se debe resaltar la labor desempeñada por personas que, de forma desinteresada, promovieron y fomentaron
FIGURA 2. Donaciones voluntaria de sangre. Perú, 19972001

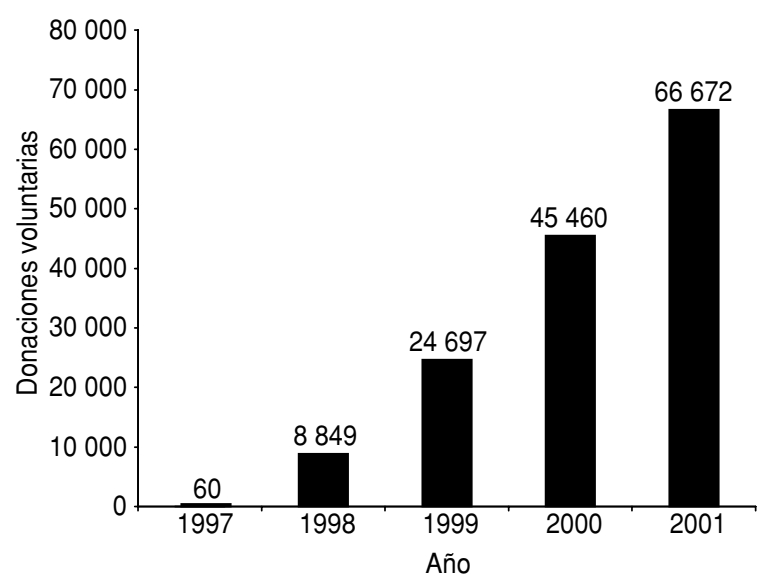

FIGURA 3. Tamizaje de las donaciones de sangre. Perú, 1997-2001

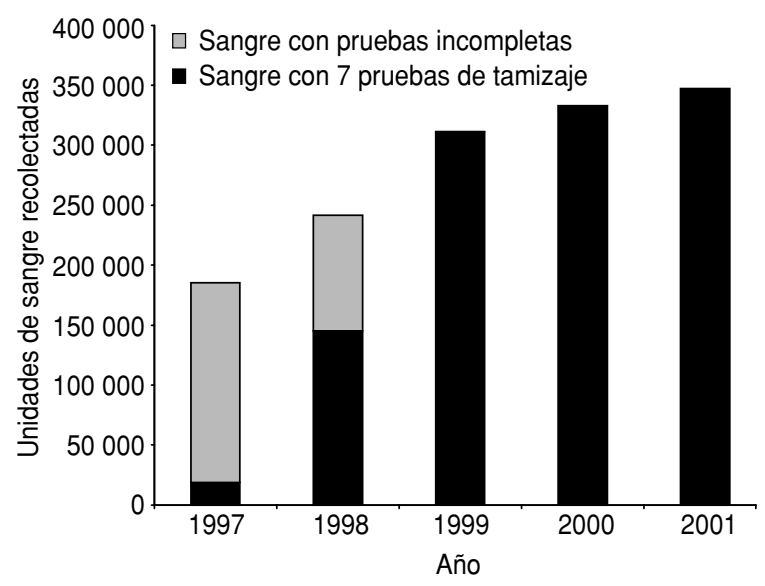

actividades tendientes a garantizar la obtención de sangre segura mediante donaciones voluntarias.

Entre los logros obtenidos hasta la fecha se pueden destacar los siguientes:

- Se ha elevado a $19,5 \%$ el total de donaciones de sangre voluntarias, gracias a la formación de 800 promotores de la donación voluntaria en todo el país y a la realización de más de 200 campañas en pro de la donación voluntaria (figura 2).

- Se ha extendido el tamizaje de sangre con siete pruebas al $100 \%$ de las unidades recolectadas en todo el país (figura 3), medida que ha permitido evitar aproximadamente 120000 casos de infección transfusional por VIH, hepatitis B 
FIGURA 4. Casos de infecciones adquiribles por sangre contaminada que se evitaron gracias al tamizaje. Perú, 1997-2001

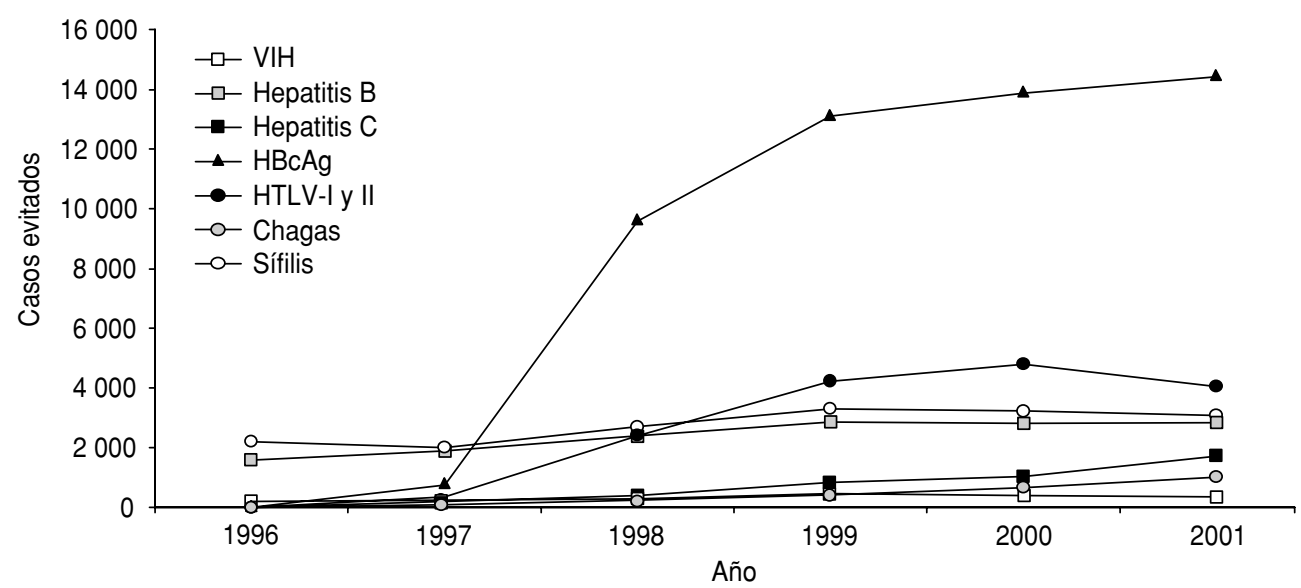

Nota: VIH: virus de la inmunodeficiencia humana; HBcAg: antígeno nuclear del virus de la hepatitis B; HTLV: virus linfotrópico de células T humanas.

y C, HTLV-I y II, sífilis y enfermedad de Chagas (figura 4).

- Se ha duplicado la disponibilidad de sangre hasta cubrir $70 \%$ de las necesidades de la población, con un incremento sustancial de las donaciones voluntarias y el fraccionamiento de $80 \%$ de toda la sangre obtenida.

- Se ha mejorado el uso apropiado de hemocomponentes y se lograron una notable disminución de las transfusiones de sangre total y un incremento sostenido del empleo de paquetes globulares y otros componentes, lo que redunda en una mayor disponibilidad de este recurso para la atención de los pacientes (figura 5).

- Se han creado redes locales y regionales de bancos de sangre, con la centralización del procesamiento de la sangre obtenida. Esto permitió disminuir los costos de producción, mejorar la seguridad y calidad de la sangre y de los hemocomponentes, y disponer de estos recursos en lugares críticos, como serían las zonas de alta mortalidad materna.

- Se ha implementado el Programa de Control de Calidad en Bancos de Sangre para inmunoserología e inmunohematología, medida que permitió detectar enfermedades transmisibles por transfusión y tipificar los grupos sanguíneos.

- Se han registrado 159 bancos de sangre, de los cuales 89 se clasificaron como Centros de Hemoterapia tipo II (lugares de obtención, procesamiento, transfusión y distribución de sangre) y 70 como Centros de Hemoterapia tipo I (lugares de almacenamiento y transfusión).

FIGURA 5. Uso de sangre total y componentes sanguíneos. Perú, 1997-2001

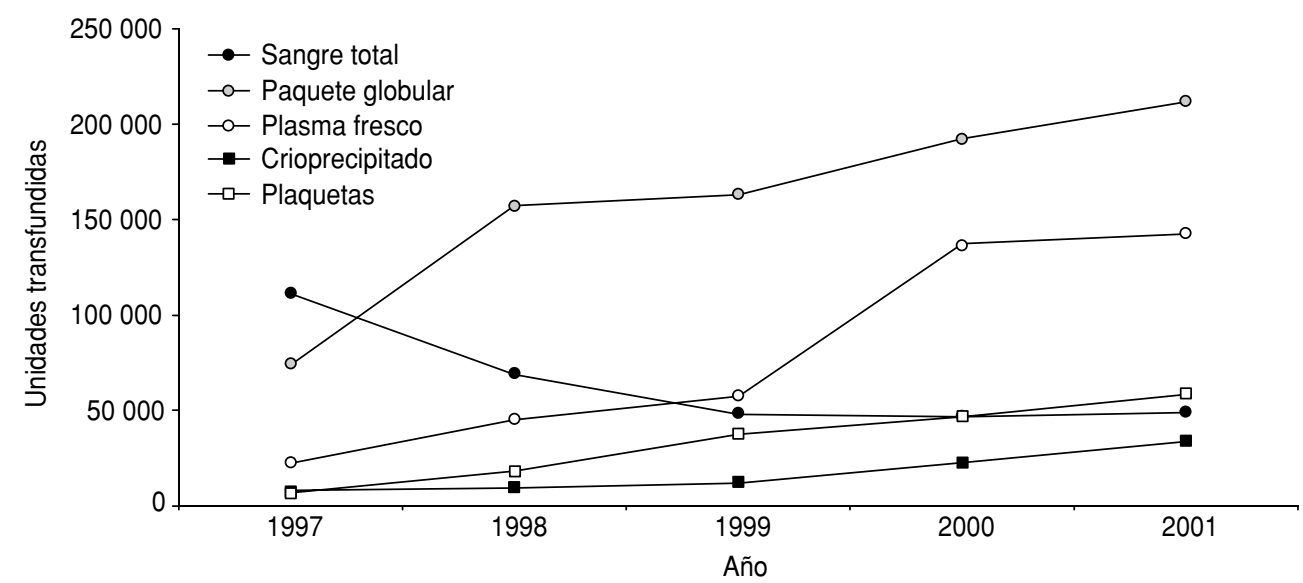


CUADRO 1. Algunas disposiciones establecidas para regular la obtención, el procesamiento y la utilización de sangre y hemocomponentes en el Perú

- R. M. 136-98-SA/DM, del 15 de abril de 1998. Establece el 23 de mayo como Día Nacional del Donante Voluntario de Sangre.

- R. M. 237-98-SA/DM, del 24 de junio de 1998. Aprueba la Doctrina, Normas y Procedimientos del PRONAHEBAS como instrumento normativo.

- R. M. 283-99-A/DM, del 10 de junio de 1999. Establece las normas de procedimientos para el control, las medidas de seguridad y las sanciones de los bancos de sangre.

- Decreto Supremo 002-99-SA, del 18 de junio de 1999. Aprueba el Texto Único de Procedimientos Administrativos del Ministerio de Salud, entre los que se encuentran los requisitos para la inscripción en el Registro Nacional de Bancos de Sangre y la Autorización Sanitaria para su funcionamiento.

- R. M. 307-99-SA/DM, del 22 de junio de 1999. Aprueba las Normas Técnicas para Proyectos de Arquitectura y Equipamiento de los Centros Hemodadores.

- R. M. 540-99-SA/DM, del 30 de octubre de 1999. Aprueba los requisitos que deben cumplir los bancos de sangre para obtener la autorización sanitaria de funcionamiento.

- Todas las unidades de hemocomponentes transfundidos, que fueron, más de 300 000, poseían el Sello Nacional de Calidad de Sangre.

- Aproximadamente 1000 trabajadores de bancos de sangre recibieron capacitación mediante los módulos "Sangre Segura" y fueron debidamente certificados.

- Se realizaron supervisiones periódicas de los bancos de sangre por parte de las direcciones de salud.

La regulación de esta actividad mediante la promulgación de leyes y normas que regulan los diferentes elementos relacionados con la obtención, procesamiento y utilización de la sangre y los hemocomponentes constituye otro de los logros obtenidos en este período (cuadro 1 ).

\section{DISCUSIÓN}

Para lograr los objetivos trazados, al inicio del Programa fue necesario sortear algunas dificultades. Unas fueron económicas; otras consistieron en convencer a las autoridades de salud de la importancia que tiene para el país poder contar con sangre segura para uso terapéutico. La alta tasa de infecciones transfusionales que se observaba hasta 1996 (1) motivó a todos los responsables a participar en la tarea de revertir esa situación. Por ello se hizo hincapié en la realización de las siete pruebas de tamizaje obligatorias y en la promoción de la donación voluntaria de sangre.

Con respecto al tamizaje de agentes infecciosos, al principio se encontró mucha reticencia por parte de los directores y administradores de hospitales debido al incremento en los costos que ello implicaba. Incluso, en algunas oportunidades hubo una resistencia abierta a cumplir estas normas sin una argumentación técnica o científica adecuada. Cuando más tarde se conocieron los problemas jurídicos que se presentaron en otros países por no poner en práctica las medidas de seguridad en torno a la sangre transfundida $(4,5)$, estos obstáculos se redujeron a un mínimo. Sin embargo, en algunos lugares el tamizaje todavía se considera innecesario y se realiza solo para cumplir con lo establecido por el Ministerio de Salud.

En cuanto a la promoción de la donación voluntaria de sangre, se desechó la práctica de esperar a que los donantes acudiesen a los bancos de sangre por obligación, con todos los riesgos que ello implicaba. La búsqueda activa de donantes voluntarios en centros de trabajo o estudio, aunque es más laboriosa, a la larga garantiza una sangre más segura y permite captar donantes voluntarios habituales, es decir, personas que acuden de forma periódica a donar sangre sin ser obligadas o motivadas por nadie. Como prueba de ello, algunos bancos de sangre - como los de los hospitales San Bartolomé, Arzobispo Loayza y Barranca, en Lima (6), o los de los hospitales regionales del Cuzco, Lambayeque y Tacna - se especializaron en desarrollar estas actividades mediante campañas o promotores de donaciones voluntarias (7).

Otro aspecto que se debe tener en cuenta es la necesidad de un marco jurídico claro que defina las obligaciones y requisitos que deben reunir los bancos de sangre para garantizar la seguridad, disponibilidad y calidad de la obtención y el uso de sangre con fines terapéuticos. Las diversas normas promulgadas por el Ministerio de Salud con posterioridad a la Ley 26454 permitieron consolidar lo establecido por esta y llenar el vacío existente hasta ese momento. Así, por ejemplo, los requisitos para la inscripción de los bancos de sangre en el Registro Nacional del Ministerio de Salud, establecidos en el Texto Único de Procedimientos Administrativos, se corresponden con los parámetros internacionales establecidos para el funcionamiento idóneo de los bancos de sangre.

Los establecimientos de salud que disponen de centros de hemoterapia debidamente certificados fueron incluidos en un Catálogo Nacional de Bancos de Sangre (8), similar al que existe en España. Ese catálogo cuenta con información sobre la ubicación geográfica (por departamentos) de estos centros, el número de unidades que cada uno procesa o utiliza al año, y la categoría (I ó II) a la que pertenece cada entidad. Con esta información es posible conocer los hospitales y clínicas que tienen bancos de sangre y que cumplen con los requisitos 
para un funcionamiento apropiado que garantice la seguridad y la calidad de su servicio.

Finalmente, la capacitación del personal de salud no solo abarca a quienes trabajan en los bancos de sangre, sino también a los médicos que administran los hemocomponentes necesarios a sus pacientes para su tratamiento eficaz y oportuno. Los términos "sangre fresca" y "sangre completa", hoy en desuso, seguían vigentes entre los profesionales médicos debido a una deficiente capacitación de pregrado o posgrado en materia de hemoterapia. Por ello se elaboró un compendio sobre el uso racional de la sangre y de los hemocomponentes (9) como fuente de referencia para los médicos que en la práctica hospitalaria necesitan una orientación definitiva y autorizada. Asimismo, la realización de cursos de hemoterapia, en colaboración con el Colegio Médico de las provincias, permitió llegar a un mayor número de profesionales de la salud, quienes actualizaron sus conocimientos para una correcta práctica en el uso de la sangre y los hemocomponentes.

El futuro está lleno de retos para los que trabajan en el campo de la hemoterapia. Estos se reflejan en las metas trazadas para esta actividad en todo el país:

1. La centralización del procesamiento de la sangre en los llamados centros "hemodadores" y la regionalización de los bancos de sangre, con la formación de redes locales y regionales, agrupadas a su vez en una red nacional. Esto permitirá mejorar la calidad de la sangre obtenida y reducir su costo, tal como han logrado países como Chile y Colombia y anteriormente, Brasil. De esta manera se podrá satisfacer las necesidades de sangre en un futuro no muy lejano y disminuir la mortalidad por falta de sangre.

2. Lograr que $50 \%$ de la sangre captada provenga de donantes voluntarios, meta fijada por la OPS para todos los países de la Región en el año 2003. Esto se podrá lograr mediante el fomento permanente de las donaciones voluntarias por promotores debidamente formados y encargados de inculcarle este concepto al resto de la población, y mediante la realización de campañas en pro de la donación de sangre. Estas campañas no deben tener por finalidad obtener sangre, sino captar donantes voluntarios habituales y demostrarle a la población que la donación no produce daño alguno.

3. La acreditación de los bancos de sangre de acuerdo con los parámetros internacionales establecidos por autoridades en este campo, como la OPS y la Asociación Estadounidense de Bancos de Sangre (AABB). Los centros de hemoterapia, ya sean del tipo I ó II, deberán satisfacer los requisitos que garantizan la obtención, el procesa- miento y el almacenamiento apropiado de la sangre y los hemocomponentes, así como su uso racional. Estos requisitos deben estar contemplados en las normas de funcionamiento emitidas por el Ministerio de Salud.

4. La capacitación permanente de recursos humanos, incluido el personal de bancos de sangre -haciendo hincapié en las técnicas de inmunohematología y de control de calidad-y otro tipo de personal de salud, especialmente médicos, mediante cursos de hemoterapia que incidan en el uso racional y apropiado de la sangre y de los hemocomponentes, así como el conocimiento del marco jurídico existente que regula la práctica de la hemoterapia en el país. Los Comités Transfusionales Intrahospitalarios son el respaldo necesario para lograr este objetivo, y una de sus funciones debe ser la de capacitar al personal de salud directamente involucrado en la obtención y el uso de la sangre con fines terapéuticos.

Agradecimientos. Les extendemos un agradecimiento muy especial a José Ramiro Cruz, Alex Indrikovs, María Dolores, Gabriel Schmunis, Ramón Kranwinkel y a todas las personas que hicieron posible mejorar la seguridad de la sangre que se transfunde en Perú.

\section{SYNOPSIS}

\section{Peru's experience with a national blood banking program}

This paper describes Peru's experiences with its National Blood Banking Program. Until the mid-1990s, the country faced a host of problems, including the lack of a legal framework to regulate blood banks, a high maternal mortality rate due to a shortage of blood, virtually no voluntary donations, a high risk of infection from transfusions, the use of only whole blood for transfusion, serious disorganization in the blood banks, deficiencies in blood bank supervision and control, no training programs, indifference on the part of health officials, frequent selling of blood, and limited community awareness.

Subsequently, a strategic plan was prepared that made it possible to solve many of those problems. Legal instruments were prepared; the rate of voluntary donations rose from $0 \%$ to $19.5 \%$; the safety of the blood was improved through compulsory screening of all donated blood units for seven markers of infectious diseases, as well as by placing a national seal of quality on all screened units. The availability of blood doubled, thus meeting $70 \%$ of the need; sales of blood decreased; and the use of blood components was improved, with $80 \%$ of the blood being fractionated. In addition, supervisory control of $100 \%$ of the blood banks in the country was achieved, a national registry was established, the cost-benefit relationship for blood units was improved through centralized screening, internal and external quality control was 
made mandatory, and pro-donation campaigns led to commitments from civil society.

While important, all these achievements represent just a first step. This is especially true given that developing the National Blood Banking Program required the participation of outside organizations, such as the Pan American Health
Organization, whose support, together with the experience provided by other countries, was key. The Program is facing a number of new challenges, and the progress that has been achieved could be threatened if current activities stagnate or if officials become complacent.

\section{REFERENCIAS}

1. Schmunis G, Zicker F, Pinheiro F, Brandling-Bennett D. Riesgo de enfermedades infecciosas transmitidas por transfusión en Centro y Sudamérica. Washington, D.C.: OPS; 1996.

2. Fuentes Rivera J, Roca O, Leiva M, Barbaran A, Maldonado F, Sakuray S, et al. Informe memoria 1997-2000 del Programa Nacional de Hemoterapia y Bancos de Sangre. Lima: Ministerio de Salud; 2001.

3. Fuentes Rivera J, Roca O, Leiva M, Barbaran A, Rodríguez Mitzi, Vidal J, et al. Doctrina, normas y procedimientos del Programa Nacional de Hemoterapia y Bancos de Sangre. Lima: Ministerio de Salud; 1998.
4. De la Cal J. Condenan al INSALUD a indemnizar a tres familias de hemofílicos muertos por suministrarles sangre contaminada. El Mundo (Madrid). 8 de noviembre de 1999

5. Soler Roca G. Problemas médico-legales de las hepatitis postransfusionales. Revista Española de Medicina Legal 1998; 22:47-49.

6. Fuentes Rivera J, Roca O, Leiva M, Barbaran A, Maldonado F, Sakuray S, et al. Boletín informativo del Programa Nacional de Hemoterapia y Bancos de Sangre. Lima: Ministerio de Salud; 1999.
7. Fuentes Rivera J, Roca $\mathrm{O}$, Hernández $\mathrm{G}$, Barbaran A, Maldonado F, Sakuray S, et al. Guía para promotores en donación voluntaria de sangre. Lima: Ministerio de Salud; 2001.

8. Fuentes Rivera J, Roca O, Leiva M, Barbaran A, Maldonado F, Sakuray S, et al. Catálogo nacional de bancos de sangre. Lima: Ministerio de Salud; 2001.

9. Fuentes Rivera J, Roca O, Aguilar L, Rodríguez M, Barbaran A, Guillén M, et al. Uso racional de sangre y hemocomponentes. Lima: Ministerio de Salud; 1998.

\section{Premio en Bioética Manuel Velasco-Suárez - 2003}

La Fundación Panamericana de Salud y Educación, en cooperación con el Gobierno de México y la Organización Panamericana de la Salud, ha creado la Beca en Bioética Manuel Velasco-Suárez en honor de este investigador y erudito méxicano, quien fundó la Comisión Nacional de Bioética de México. La beca, que consiste en US\$ 10000 en efectivo, un Certificado de Honor y un viaje a Washington, D.C., Estados Unidos de América, se le otorga a un académico o investigador latinoamericano 0 caribeño menor de 41 años con un título de doctorado 0 su equivalente que presente una propuesta sobresaliente de actividades académicas en las áreas de bioética básica o aplicada para el año de usufructo de la beca. Las candidaturas deberán ser presentadas por un representante oficial de la institución académica a la que pertenece el candidato y deberán acompañarse de la propuesta de trabajo del solicitante más un breve relato de su trabajo previo.

La fecha límite para la presentación de solicitudes es el 15 de junio de 2003.

Información:

Premio en Bioética Manuel Velasco-Suárez

Fundación Panamericana de la Salud y Educación 525 23rd Street N.W.

Washington, D.C. 20037

Tel.: (202) 974-3416 • Fax: (202) 974-3636

Correo electrónico: pahef@paho.org 